\title{
Impact of Educational Intervention on Preventive Strategies for Cardiovascular Disease Risk Modification among Patients with Type II Diabetes Mellitus
}

\author{
Darshana Desai ${ }^{1 *}$, Ipseeta Ray Mohanty ${ }^{1}$ and Sandeep Rai ${ }^{2}$ \\ ${ }^{1}$ Department of Pharmacology, MGM Medical College, India \\ ${ }^{2}$ Department of Medicine, MGM Medical College, India
}

Submission: August 07, 2020; Published: September 17, 2020

*Corresponding author: Ipseeta Ray Mohanty, Department of Pharmacology, MGM Medical College, Kamothe, Navi Mumbai, Maharashtra, 410209, India

Abstract

Objective: The Type II Diabetes mellitus (DM) patients were stratified into CVD risk categories. The lifestyle and prescription pattern of DM patients was studied. Also, impact of educational intervention on preventive strategies for CVD risk modification was assessed.

Methods: The WHO Cardiovascular disease (CVD) risk prediction charts were used to categorize DM into various CVD risk categories and were imparted education intervention based on WHO Guidelines for Assessment and Management of CVD Risk.

Results: $67.5 \%$ of DM had low, $15 \%$ medium, $15 \%$ high and $2.5 \%$ had very high CVD risk. $100 \%$ of DM patients with high CVD risk profile were on antihypertensive and antiplatelet drugs though only $50 \%$ were on hypolipidemic agents. Educational intervention improved the knowledge regarding preventive strategies for CVD Risk modification \{weight control $(\mathrm{p}<0.05)$, glycemic control $(\mathrm{P}<0.01)$, benefits of hypolipidemic agents $(\mathrm{p}<0.01)$ and antiplatelet drug aspirin $(\mathrm{P}<0.001)$ usage $\}$.

Conclusion: $32.5 \%$ Type 2 diabetic patients have medium to very high risk of developing a cardiovascular event. There is a scope to increase the prescription of hypolipidemic drugs for CVD risk modification. Educational intervention resulted in a significant increase in knowledge regarding preventive strategies for CVD Risk modification.

Keywords: Cardiovascular disease; Risk prediction charts; Diabetes mellitus; World health Organization

Abbreviations: CVD: Cardiovascular disease; DM: Type II Diabetes mellitus; WHO: World Health Organization; ICMR: Indian Council of Medical Research

\section{Introduction}

Cardiovascular disease (CVD) is the major cause of death in patients with type 2 diabetes mellitus (DM), as majority of DM patients' die of myocardial infarction (MI) or stroke, and an even greater proportion of patients have serious burdensome complications [1-3]. Recent large clinical trials, the Action in Diabetes and Vascular Disease (ADVANCE), Action to Control Cardiovascular Risk in Diabetes (ACCORD) reported no significant decrease in primary cardiovascular endpoints with solely intensive glu cose control as besides blood glucose, other variables: age, gender, high blood pressure, smoking and dyslipidemia are the other major contributing risk factors for developing CVD. Thus, besides good glycemic control comprehensive risk factor management, including blood pressure control, lipid management, weight reduction in overweight or obese individuals, and smoking cessation, are also needed [4-10].

Therefore, development of strategies for the control of CVD risk factors and primary prevention of cardiovascular complications in diabetes remains the most important clinical objective. To address this issue, various multivariable CVD risk prediction algorithms such as Framingham Risk score, UKPDS Risk Engine, WHO charts have been developed that can be used by primary care physicians to assess in individual patients the risk of developing of CVD [4]. Risk factor modification can reduce clinical events and premature death in people with established cardiovascular disease as well as in those who are at high cardiovascular risk due to one or more risk factors. 


\section{Current Research in Diabetes \& Obesity Journal}

Several studies including WHO Guidelines for assessment and management of CVD risk have shown that besides antidiabetic drugs: antihypertensive hypolipidemic, antiplatelet drugs are the pharmacological agents that can be prescribed for CVD risk modification based on the individuals CVD risk profile. Additionally, non-pharmacological recommendations focusing on diet, smoking cessation and regular physical activity will provide guidance on self-management strategies to DM patients (with risk factors who have not yet developed clinically manifest cardiovascular disease) to prevent adverse CVD outcomes in the future (primary prevention).

With this point of view the present study was designed. The primary objective was to use the WHO/ISH CVD risk prediction charts to categorize the DM patients as having very high, high, medium and low CVD risk. The WHO/ISH risk prediction charts indicate 10-year risk of a fatal or nonfatal major cardiovascular event (myocardial infarction or stroke), according to age, sex, blood pressure, smoking status, total blood cholesterol and presence or absence of diabetes mellitus.

The patient profile, cardiovascular risk factors and prescription pattern among diabetic patients of various CVD risk categories was determined. Results would provide baseline information based on which future intervention can be planned to mitigate CVD risk among diabetic patients by following the WHO Pharmacological and Non-Pharmacological recommendation for CVD risk modification.

Also, impact of educational intervention on preventive strategies for cardiovascular disease risk modification (Knowledge regarding Tobacco, Alcohol usage, Diet, Physical Activity, Weight control, Psychosocial factors, Multiple risk factor interventions) was assessed.

\section{Materials and Methods}

Necessary approval from the Institutional Ethics Committee was obtained before initiating the study.

a) Study design: Prospective, Interventional

b) Study site: Department of Pharmacology and Medicine, MGM Medical College, Kamothe, Navi Mumbai

c) Sample size: Approximately 160 patients with type II Diabetes mellitus attending the Medicine OPD were enrolled in the study.

d) Study Population: Type II diabetes mellitus patients who conformed to the specified Inclusion and Exclusion Criteria were enrolled for the study.

\section{e) Inclusion criteria:}

Aged $\geq 18$ years of age who were diagnosed with Type II DM

Patients on the Pharmacological agents for CVD risk modification for at least six months.

\section{Patients who agree to sign the consent form}

\section{f) Exclusion criteria:}

Patients with Type 1 diabetes mellitus

Pregnant women, including those with gestational diabetes

People with established coronary heart disease (CHD), cerebrovascular disease (CeVD) and peripheral vascular disease

Patients who could not complete the questionnaire

g) Study Group: Type II diabetic patients meeting the Inclusion and Exclusion criteria.

\section{Case Record Form (CRF)}

A CRF was prepared to record the following information on the Type II diabetes patient attending the medicine outdoor department.

a. Patient Profile: Age, Gender, BMI and History of Smoking Intake was noted

b. Disease Profile: Fasting and post prandial blood sugar values, duration of diabetes $(5,5-10,>10$ years), Age of onset of diabetes $(<50,50-59,>60$ years), presence of hypertension (BP recordings), presence of dyslipidemia (Lipid Profile ( $\mathrm{CH}<180 \mathrm{mg} /$ dl, HDL<45 mg/dl (Male), < 50 mg/dl (Female), LDL: >100 mg/dl and $\mathrm{TG}>150 \mathrm{mg} / \mathrm{dl}$ were recorded

c. Treatment Modality: The various drugs (Anti-diabetic, Antihypertensive, Anti-platelet and Hypolipidemic agents) prescribed for cardiovascular disease modification were noted.

d. WHO Risk Prediction Chart for Assessment and Management of CVD Risk

World Health Organization/International Society of Hypertension (WHO/ISH) risk prediction chart was used for the estimation of total cardiovascular risk of DM patients.

\section{Study Procedure}

The above-mentioned data and completed prescriptions were collected on predesigned case record form. All the diabetic patients attending the medicine outdoor department were screened and those eligible as per the inclusion and exclusion criteria were enrolled. Written informed consent was obtained from each patient. Subsequently the prescription details and the parameters mentioned on the CRF were filled up. The WHO Risk Prediction Chart for Assessment and Management of CVD Risk was used to stratify the patients into risk categories (Very high, high, medium and low). The patient's baseline knowledge on Preventive Strategies for CVD Risk modification was scored. Subsequently, the patients were counselled based on WHO CVD Risk Reduction Guidelines and thereafter re-administered the same questionnaire to assess the impact of structured counselling. The data was collected; analysed and appropriate statistics was applied to obtain meaningful information. 


\section{Current Research in Diabetes \& Obesity Journal}

\section{Data Analysis and Statistical Methods}

Descriptive statistics was used to summarize patients' demographics and survey responses. Logistic regression models were used to assess the relationship between high CVD risk and potential influencing factors. $\mathrm{P}<0.05$ was considered as statistically significant.

\section{Results}

\section{Disposition summary of type II diabetic patients}

One hundred sixty (160) diabetic patients who attended the medicine diabetic OPD received structured educational intervention for cardiovascular disease risk modification (Figure 1).

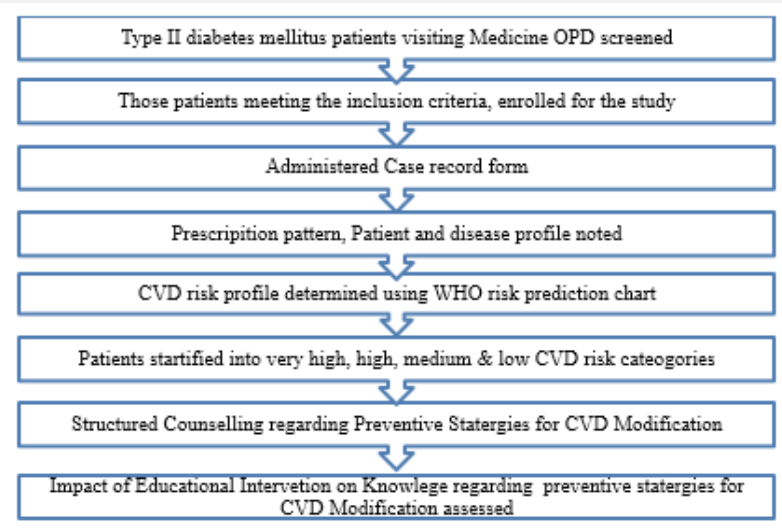

Figure 1: Disposition summary of Type II diabetic patients.

\section{Patient profile of type II diabetic subjects}

The age distribution of the Type II diabetic patients who were counselled for cardiovascular risk modification is depicted in
Table 1. Maximum (38.75 \%) Type II diabetic patients were of the age group 50-59 years. Male/Female ratio was found to be 1.3. It was found that $16.3 \%$ patients were obese (Table 1 ).

Table 1: Characteristics of type II diabetic patients enrolled for the study $(N=160)$.

\begin{tabular}{|c|c|c|c|}
\hline S. No & & $\mathbf{N = 1 6 0}$ & Percentage (100\%) \\
\hline $\mathbf{1}$ & Age & 58 & $36.25 \%$ \\
\hline & $<50$ & 62 & $38.75 \%$ \\
\hline & $50-59$ & 38 & $23.75 \%$ \\
\hline $\mathbf{2}$ & $60-69$ & 2 & $1.25 \%$ \\
\hline & $>70$ & & $56.25 \%$ \\
\hline $\mathbf{3}$ & Gender & 90 & $43.75 \%$ \\
\hline & Male & 70 & $57.50 \%$ \\
\hline & Female & & $43.75 \%$ \\
\hline
\end{tabular}

\section{Disease profile among type II diabetic subjects}

Disease profile of the Type II diabetic patients who were counselled for cardiovascular risk modification is depicted in Table 2. Maximum (68.75 \%) type 2 diabetic patients had diabetes for more for more than 7 years. Hypertension was encountered among $22.5 \%$ patients and Dyslipidemia among $13.75 \%$ patients. It was found that $52.5 \%$ patients had uncontrolled glycemic control and $33 \%$ had uncontrolled hypertension. Additional characteristics of these Type II diabetic subjects are presented in Table 2.

\section{Cardiovascular risk stratification among Type II diabetic patients}

Patients were stratified into cardiovascular risk categories. Out of the type 2 diabetic patients screened for CVD risk, $67.5 \%$ 


\section{Current Research in Diabetes \& Obesity Journal}

has low CVD risk ( $<10 \%$ scores), $15 \%$ medium CVD risk (10 to \% were categorized as having very high CVD risk ( $>30 \%$ scores) $20 \%$ scores), $15 \%$ high CVD risk (20 to $30 \%$ scores) and 2.5 (Figure 2).

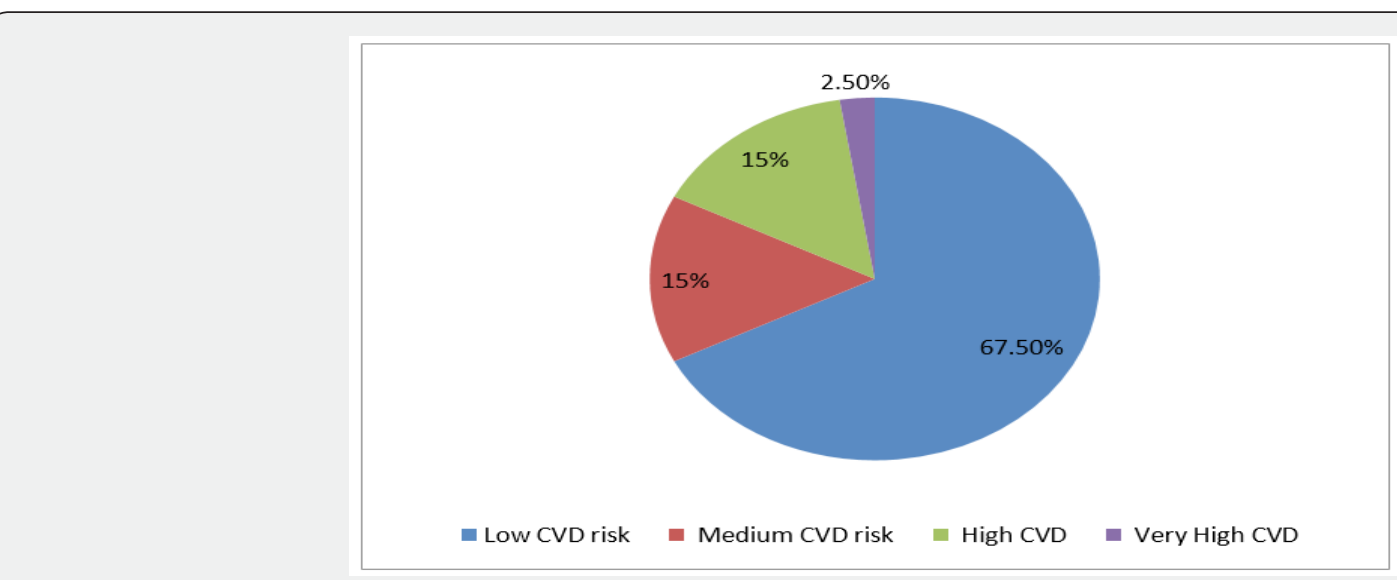

Figure 2: Cardiovascular Risk Stratification among Type II diabetic patients.

\section{Patient profile based on CVD risk profile}

Maximum patients with medium, high and very high CVD risk belonged to the age group 60-69 years. The gender distribution and characteristics of the Type 2 diabetic patients of various risk categories is depicted in table 3.

Table 2: Patient profile of Type II diabetic patients enrolled for the study.

\begin{tabular}{|c|c|c|c|}
\hline S.No & & $\mathbf{N}$ & Percentage \\
\hline \multicolumn{4}{|c|}{ Diabetes } \\
\hline \multirow[t]{3}{*}{$\mathrm{A}$} & Duration of diabetes & & \\
\hline & $>7$ years & 50 & $31.25 \%$ \\
\hline & $<7$ years & 110 & $68.75 \%$ \\
\hline \multirow[t]{3}{*}{$\mathrm{B}$} & Glycemic Control & & \\
\hline & Controlled & 76 & $47.50 \%$ \\
\hline & Uncontrolled & 84 & $52.50 \%$ \\
\hline \multicolumn{4}{|c|}{ Hypertension } \\
\hline \multirow[t]{3}{*}{$\mathrm{A}$} & Presence of Hypertension & 36 & $22.50 \%$ \\
\hline & Controlled & 24 & $66.60 \%$ \\
\hline & Uncontrolled & 12 & $33.30 \%$ \\
\hline B & Family History & 82 & $51.25 \%$ \\
\hline \multicolumn{4}{|c|}{ Dyslipidemia } \\
\hline & Presence of Dyslipidemia & 22 & $13.75 \%$ \\
\hline
\end{tabular}

Table 3: Patient Profile based on CVD Risk Profile.

\begin{tabular}{|c|c|c|c|c|c|}
\hline S. No & & Low CVD Risk N=108 & $\begin{array}{c}\text { Medium CVD Risk N (\%) } \\
\mathbf{N = 2 4}\end{array}$ & $\begin{array}{c}\text { High CVD Risk N (\%) } \\
\text { N=24 }\end{array}$ & $\begin{array}{c}\text { Very High CVD Risk N (\%) } \\
\text { N=04 }\end{array}$ \\
\hline $\mathbf{1}$ & Age & & & & 0 \\
\hline & $<50$ & $54(50 \%)$ & $4(16.6 \%)$ & $2(8.3 \%)$ & 0 \\
\hline & $50-59$ & $54(50 \%)$ & $6(25 \%)$ & $20(83.3 \%)$ & $4(100 \%)$ \\
\hline & $60-69$ & 0 & $14(58.3 \%)$ & $2(8.3 \%)$ & 0 \\
\hline & $>70$ & 0 & 0 & & \\
\hline
\end{tabular}




\section{Current Research in Diabetes \& Obesity Journal}

\begin{tabular}{|c|c|c|c|c|c|}
\hline & Male & $40(38.4 \%)$ & $24(100 \%)$ & $18(75 \%)$ & $4(100 \%)$ \\
\hline & Female & $64(61.6 \%)$ & 0 & $6(25 \%)$ & 0 \\
\hline $\mathbf{3}$ & BMI & & & & \\
\hline & Obese & $8(7.74 \%)$ & $6(25 \%)$ & $8(33.3 \%)$ & $4(100 \%)$ \\
\hline
\end{tabular}

\section{Disease profile based on CVD risk profile}

Duration of diabetes more than 7 years (long standing diabetes) was encountered in $75 \%$ belonging to medium CVD risk, $53.3 \%$ high CVD risk and $50 \%$ of very high CVD risk. Poor glycemic control was noted among $66.6 \%$ belonging to medium CVD risk, $75 \%$ high CVD risk and $100 \%$ of very high CVD risk. Hypertension was encountered in $50 \%$ and $100 \%$ of the type 2 diabetic patients and dyslipidemia among $33.3 \%$ and $100 \%$ respectively with high and very high CVD risk (Figure 3).

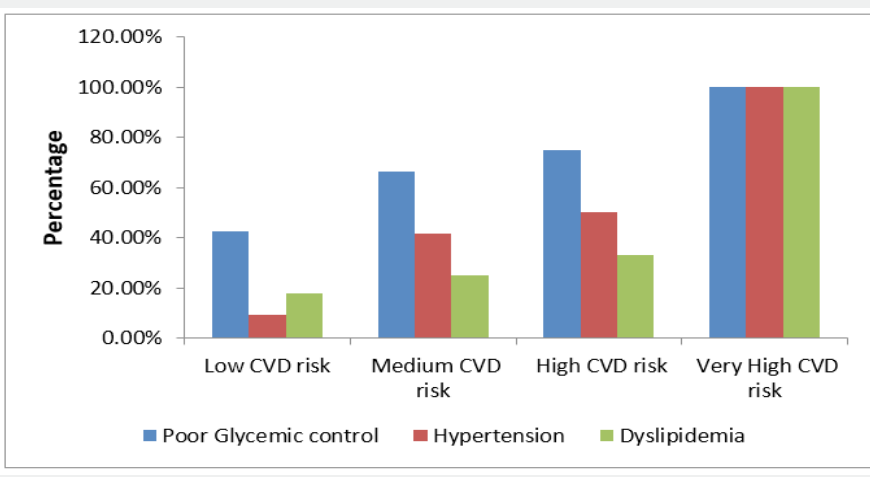

Figure 3: Risk factors present in patients based on CVD Risk Profile.

\section{Drugs used for cardiovascular disease risk modification based on CVD Risk Profile}

Antihypertensive drugs were prescribed among $41.6 \%$ diabetic patients with medium CVD risk, $50 \%$ with high CVD risk and $100 \%$ of very high CVD risk. Antiplatelet drug was prescribed among $100 \%$ of diabetic patients with very high CVD risk. Hypolipidemic drug prescription was encountered among 25\% and $50 \%$ of the diabetic patients with high and very high CVD risk (Table 4).

Table 4: Drugs used for cardiovascular disease risk modification based on CVD Risk Profile.

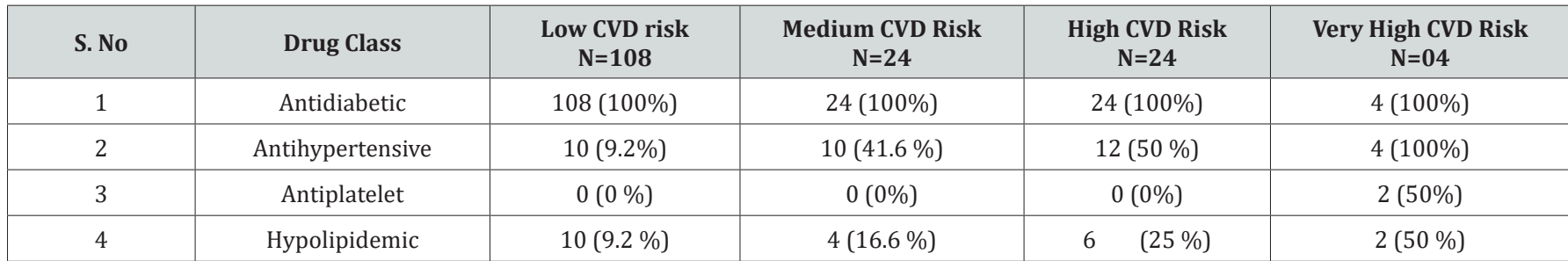

Impact of counselling on knowledge related to risk factors associated with cardiovascular disease

The WHO Risk Prediction Chart for Assessment and Management of CVD Risk was used to stratify the patients into risk categories (Very high, high, medium and low). The patient's baseline knowledge on Preventive Strategies for CVD Risk modification was scored. Subsequently, the patients were counselled based on WHO CVD Risk Reduction Guidelines and thereafter re-administered the same questionnaire to assess the impact of structured counselling. Counselling resulted in a significant increase in knowledge regarding preventive Strategies for CVD Risk modification. The baseline total knowledge score increased significantly related to items: body weight $(\mathrm{p}<0.05)$, glycemic control $(\mathrm{P}<0.01)$, benefits of hypolipidemic agents $(\mathrm{p}<0.01)$ and antiplatelet drug aspirin $(\mathrm{P}<0.001)$ usage (Figure 4$)$. Majority of the type 2 diabetic patients found the counselling to be useful, complete and balanced.

\section{Discussion}

Diabetes is fast gaining the status of a potential epidemic in India with more than 62 million diabetic individuals currently diagnosed with the disease [11-13]. In 2000, India (31.7 million) 


\section{Current Research in Diabetes \& Obesity Journal}

topped the world with the highest number of people with diabetes mellitus. Cardiovascular disease (CVD) is the major cause of death in patients with type 2 diabetes mellitus (DM) [14]. The myriad of cardiovascular complications associated with diabetes will make the disease a public health emergency in the future [1].

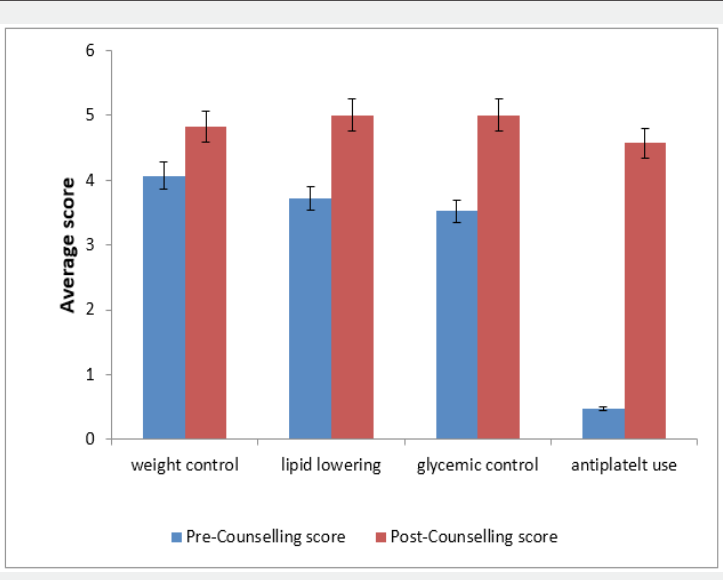

Figure 4: Impact of Educational Intervention on knowledge related to CVD prevention strategies.

Among diabetic patients, the impact of sole glucose lowering on cardiovascular complications is a hotly debated issue. The United Kingdom Prospective Diabetes Study (UKPDS) was the first clinical trial to provide key evidence of the importance of using intensive therapy for diabetes control in individuals with newly diagnosed DM. Recent large clinical trials, the Action in Diabetes and Vascular Disease (ADVANCE), Action to Control Cardiovascular Risk in Diabetes (ACCORD), and the Veterans Affairs Diabetes Trial (VADT), reported no significant decrease in primary cardiovascular endpoints with intensive glucose control [15-17]. Therefore, glycemic control is not the only factor to be considered to decrease the risks of cardiovascular complications.

It is widely accepted that besides blood glucose, other variables: age, gender, high blood pressure, smoking and dyslipidemia are the major risk factors for developing CVD. Thus, comprehensive risk factor management, including blood pressure control, lipid management, weight reduction in overweight or obese individuals, and smoking cessation, are also needed. The results of the ACCORD and ADVANCE studies and the VADT should be interpreted in the context of comprehensive care of patients with diabetes [18]. Therefore, Interventions for simultaneous optimal control of co-morbidities often present in DM, such as hypertension and hyperlipidemia, have been shown to be a more effective strategy in reducing cardiovascular risk than targeting only blood glucose levels per se.

Several large randomized trials and systematic reviews have shown the benefit of drugs such as statins in patients with high cardiovascular risk. Anti-platelets are capable of reducing major cardiovascular events, and many antihypertensive drugs, mainly angiotensin-converting enzyme inhibitors (ACEI), have the potential to reduce cardiovascular events. Additionally, non- pharmacological interventions, such as cardioprotective diets, smoking cessation and regular physical activity, although the data are less robust than those in studies with pharmacological therapies, are also associated with a reduction in clinically relevant CVD outcomes [19].

Thus, it is critical that therapy should also be directed towards aggressive modification of the cardiovascular risk factors in diabetic patients. Therefore development of strategies for the primary prevention of cardiovascular complications in diabetes remains the most important clinical objective. To address this issue, various multivariable CVD risk prediction algorithms such as Framingham Risk score, UKPDS Risk Engine, WHO charts have been developed incorporating these risk factors, that can be used by primary care physicians to assess in individual patients the risk of developing of CVD, ie, coronary heart disease, stroke, peripheral vascular disease, or heart failure [20]. Risk factor modification can reduce clinical events and premature death in people with established cardiovascular disease as well as in those who are at high cardiovascular risk due to one or more risk factors.

The estimated absolute CVD event rates predicted based on Prediction tools can be used to quantify risk and to guide preventive primary care among DM patients. Individuals with a high global CVD risk scores (eg, a 10-year risk of a CVD event (coronary, cerebrovascular, and peripheral artery disease and heart failure) more than $20 \%$ ) require more aggressive risk factor modification. The goal of therapy of dyslipidemia, diabetes, and hypertension should be linked to the global CVD risk. In the future the use of CV risk prediction tools may be used to estimate overall $\mathrm{CV}$ risk in order to decide on treatment. When resources are limited, individual counselling and provision of care may have to be prioritized according to cardiovascular risk [21]. 


\section{Current Research in Diabetes \& Obesity Journal}

With this point of view the present study was designed. The WHO CVD risk prediction charts were used to categorize the DM patients as having very high, high, medium and low CVD risk. The patient profile, cardiovascular risk factors and prescription pattern among diabetic patients of various CVD risk categories was determined. Also, impact of structured counselling on preventive strategies for cardiovascular disease risk modification was assessed.

Maximum Type II diabetic patients who were counselled for cardiovascular risk modification were of the age group 5059 years. A large proportion of the type 2 diabetic patients had long standing diabetes for more than 7 years. Hypertension was encountered among $22.5 \%$ patients and dyslipidemia among 13.75 $\%$ patients. It was found that $52.5 \%$ patients had uncontrolled glycemic control and $33 \%$ had uncontrolled hypertension. Studies have shown that uncontrolled glycaemia and hypertension are the major culprits precipitating a cardiovascular event such as myocardial infarction and stroke. Therefore, efforts should be directed to keep blood sugar and blood pressure under clinically permissible limits.

WHO Guidelines for CVD Prevention recommends the use of antihypertensive, hypolipidemic and antiplatelet drugs besides antidiabetic drugs for CVD risk modification $[4,10]$. The drug therapy of dyslipidemia, diabetes, and hypertension should be linked to the global CVD risk. In the present study it was found that for the management of diabetes maximum (97.5\%) type 2 diabetic patients were prescribed oral hypoglycemic agents alone. Aspirin was prescribed only in $1.25 \%$ cases and hypolipidemic agents (statins) among $13.75 \%$ type II diabetic patients.

The WHO Risk Prediction Chart for Assessment and Management of CVD Risk was used to stratify the patients into risk categories (Very high, high, medium and low) [4]. Out of the type 2 diabetic patients screened for CVD risk, $67.5 \%$ has low CVD risk ( $<10 \%$ scores), $15 \%$ medium CVD risk (10 to $20 \%$ scores), 15 $\%$ high CVD risk (20 to $30 \%$ scores) and $2.5 \%$ were categorized as having very high CVD risk ( $>30 \%$ scores). Thus $32.5 \%$ of the diabetic patients have medium to very high risk of developing a cardiovascular event in the near future. Therefore, these diabetic patients need to be monitored more closely and an intervention to modify these CVD risk factors needs to be addressed.

The prevalence of WHO/ISH "high CVD risk" ( $\geq 20 \%$ chance of developing a cardiovascular event over 10 years) was studied in Mongolia, Malaysia and Cambodia. Results showed that majority of people in all three countries has a low $(<10 \%)$ 10-year CVD risk ranging from $89.6 \%$ in Mongolia to $94.4 \%$ in Malaysia to $97 \%$ in Cambodia. The percentage of population at a high CVD risk ( $\geq 20 \%$ ) was the greatest at $6 \%$ in Mongolia, compared with $2.3 \%$ in Malaysia and $1.3 \%$ in Cambodia. In all the three countries, a higher proportion of men had moderate or high total CVD risk than women, though in none of the countries were the differences statistically significant, and the risk among both women and men increased significantly with age [10].

Duration of diabetes more than 7 years (long standing diabetes) was encountered in $75 \%$ belonging to medium CVD risk, $53.3 \%$ high CVD risk and $50 \%$ of very high CVD risk. Poor glycemic control was noted among $66.6 \%$ belonging to medium CVD risk, $75 \%$ high CVD risk and $100 \%$ of very high CVD risk. Hypertension was encountered in $50 \%$ and $100 \%$ of the type 2 diabetic patients and dyslipidemia among $33.3 \%$ and $100 \%$ respectively with high and very high CVD risk. Thus the various CVD risk factors such as poor glycemic control, hypertension, dyslipidemia was encountered in majority of the diabetic patients belonging to high and very high CVD risk profile.

Drug used for the cardiovascular risk modification among the various CVD risk categories was also assessed. It was found that antihypertensive drugs were prescribed among $41.6 \%$ diabetic patients with medium CVD risk, $50 \%$ with high CVD risk and $100 \%$ of very high CVD risk. Antiplatelet drug was prescribed among $100 \%$ of diabetic patients with very high CVD risk. Hypolipidemic drug prescription was encountered among 25\% and $50 \%$ of the diabetic patients with high and very high CVD risk. The WHO Guidelines for Assessment and Management of CVD Risk recommends the use of various classes' drugs for CVD risk modification depending upon the risk category. Those with higher CVD risk need to be treated more aggressively. Thus, there exists a scope to increase the usage of hypolipidemic drugs in patients with high and very high CVD risk category.

Reducing cigarette smoking, body weight, blood pressure, blood cholesterol, and blood glucose all have a beneficial impact on major biological cardiovascular risk factors [22-24]. Behaviors such as stopping smoking, taking regular physical activity and eating a healthy diet promote health and have no known harmful effects. They also improve the sense of well-being and are usually less expensive to the health care system than drug treatments, which may also have adverse effects. Observational studies have found that other behavioral modifications, in particular cessation of smoking, are associated with a reduction in cardiovascular disease mortality [25]. In men in the United Kingdom, a healthy lifestyle and increased physical activity have been shown to reduce the chances of developing cardiovascular disease. Therefore, interventions targeted at individuals could be expected to bring about behavioural changes and modify the CVD risk factors.

The type II diabetic patients were imparted educational intervention based on WHO Guidelines for Assessment and Management of CVD Risk on Knowledge regarding Tobacco, Alcohol usage, Diet, Physical Activity, Weight control, Psychosocial factors and Multiple risk factor interventions $[4,10]$. The patient's knowledge on Preventive Strategies for CVD Risk modification was scored. Subsequently, the patients were counselled based on WHO CVD Risk Reduction Guidelines and thereafter re- 


\section{Current Research in Diabetes \& Obesity Journal}

administered the same questionnaire to assess the impact of structured counselling. The knowledge pre and post educational intervention was compared among type II diabetic patients to assess the impact of structured counselling.

Counselling resulted in a significant increase in knowledge regarding preventive Strategies for CVD Risk modification. The baseline total knowledge score increased significantly related to items: weight control $(\mathrm{p}<0.05)$, glycemic control $(\mathrm{P}<0.01)$, benefits of hypolipidemic agents $(\mathrm{p}<0.01)$ and antiplatelet drug aspirin $(\mathrm{P}<0.001)$ usage. Majority of the type 2 diabetic patients found the counselling to be useful, complete and balanced. If knowledge related to benefits of weight control and maintaining good glycemic control translates to self-corrective behavior, it would be very beneficial in modifying the preventable CVD risk factors.

Results of the present study have provided baseline information on the CVD risk profile, drug therapy, disease, risk factor profile among diabetic patients with variable CVD risk profile. The purpose of emphasizing the recommendations elaborated in the WHO guidelines through structured counselling is to motivate and assist high-risk diabetic individuals to lower their cardiovascular risk by: quitting tobacco use, making healthy food choice, being physically active, reducing body mass index, lowering blood pressure, lowering blood cholesterol, controlling glycaemia. Data will provide future guidance on specific preventive actions to initiate at the level of patient, physician and Institute for CVD risk modification.

\section{Conclusion}

WHO CVD risk prediction charts demonstrated that one third of the Type 2 diabetic patients have medium to very high risk of developing a cardiovascular event in the near future. Education intervention resulted in a significant increase in knowledge \{related to weight control, glycemic control, benefits of hypolipidemic agents and antiplatelet drug aspirin usage\} regarding preventive strategies for CVD Risk modification.

\section{Funding}

This work was supported by Indian Council of Medical Research (ICMR), Government of India, as a Short-Term Studentship (ICMRSTS) 2017 Project (Reference ID- No. 2017-01905).

\section{References}

1. Mohan V, Venkatraman JV, Pradeepa R (2010) Epidemiology of Cardiovascular Disease in Type 2 Diabetes: The Indian Scenario. J Diabetes Sci Technol 4(1): 158-170.

2. Otgontuya D, Oum S, Buckley BS, Bonita R (2013) Assessment of total cardiovascular risk using WHO/ISH risk prediction charts in three low and middle income countries in Asia. BMC Public Health 13: 539.

3. The ADVANCE Collaborative Group (2008) Intensive blood glucose control and vascular outcomes inpatients with type 2 Diabetes. N Engl J Med 358(24): 2560-2572.
4. Prevention of Cardiovascular Disease (2007) Guidelines for assessment and management of cardiovascular risk. Geneva, World Health Organization.

5. Kaveeshwar S (2014) The current state of diabetes mellitus in India. Australasian Medical Journal 7(1): 45-48.

6. Lorber D (2014) Importance of cardiovascular disease risk management in patients with type 2 diabetes mellitus. Diabetes, Metabolic Syndrome and Obesity: Targets and Therapy 7: 169-183.

7. Kalofoutis C, Piperi C, Zisaki A, Singh J, Harris F, et al. (2006) Differences in Expression of Cardiovascular Risk Factors among Type 2 Diabetes Mellitus Patients of Different Age. Annals of the New York Academy of Sciences 1084(1): 166-177.

8. Bitton A, Gaziano T (2010) The Framingham Heart Study's Impact on Global Risk Assessment. Progress in Cardiovascular Diseases 53(1): 68-78.

9. Simmons R, Coleman R, Price H, Holman R, Khaw K, Wareham N (2008) Performance of the UK Prospective Diabetes Study Risk Engine and the Framingham Risk Equations in Estimating Cardiovascular Disease in the EPIC- Norfolk Cohort. Diabetes Care 32(4): 708-713.

10. D’ Agostino R, Vasan R, Pencina M, Wolf P, Cobain M, Massaro J (2008) General Cardiovascular Risk Profile for Use in Primary Care: The Framingham Heart Study. Circulation 117(6): 743-753.

11. Kumar A, Goel MK, Jain RB, Khanna P, Chaudhary V (2013) India towards diabetes control: Key issues. Australas Med J 6(10): 524-531.

12. Giorgino F, Leonardini A, Laviola L (2013) Cardiovascular disease and glycemic control in type 2 diabetes: now that the dust is settling from large clinical trials. Ann. N.Y. Acad. Sci 1281(1): 36-50.

13. The Action to Control Cardiovascular Risk in Diabetes Study Group (2008) Effects of intensive glucose lowering in type 2 Diabetes. N Engl J Med 358: 2545-2559.

14. Berwanger O, Alberto Piva L, Vilela Martin F (2013) Evidence-Based Therapy Prescription in High-Cardiovascular Risk Patients: The REACT Study. Arq Bras Cardiol 100(3): 212-220.

15. Fox CS, S Coady, PD Sorlie (2007) Increasing cardiovascular disease burden due to diabetes mellitus: the Framingham Heart Study. Circulation 115(12): 1544-1550.

16. Effect of intensive blood glucose control with metformin on complications in overweight patients with type 2 diabetes (UKPDS 34). UK Prospective Diabetes Study (UKPDS) Group. (1998) Lancet 352: 854-865.

17. Yusuf S (2004) Effect of potentially modifiable risk factors associated with myocardial infarction in 52 countries (the INTERHEART study): case-control study. Lancet 364(9438): 937-952.

18. Jackson R, Lynch J, Harper S (2006) Preventing coronary heart disease. BMJ 332(7542): 617-618.

19. De Backer G (2003) Third Joint Task Force of European and Other Societies on Cardiovascular Disease Prevention in Clinical Practice. European guidelines on cardiovascular disease prevention in clinical practice. Eur Heart J 24(17): 1601-1610.

20. (2003) Diet, nutrition and the prevention of chronic diseases: Report of a joint WHO/FAO expert consultation. Geneva. World Health Organization, (WHO Technical Report Series No. 916).

21. Pearson TA (2002) AHA guidelines for primary prevention of cardiovascular disease and stroke. Consensus panel guide to comprehensive risk reduction for adult patients without coronary or other atherosclerotic vascular diseases. American Heart Association Science Advisory and Coordinating Committee. Circulation 106(3): 388-391. 


\section{Current Research in Diabetes \& Obesity Journal}

22. Kawachi I, G A Colditz, M J Stampfer, W C Willett, J E Manson, et al (1994) Smoking cessation and time course of decreased risks of coronary heart disease in middleaged women. Arch Intern Med 154(2): 169-175.

23. Lam TH, Zhi Bin Li, Sai Yin Ho, Wai Man Chan, Kin Sang Ho, et al. (2002) Smoking, quitting, and mortality in a Chinese cohort of retired men. Ann Epidemiol 12(5): 316-320.
24. Godtfredsen NS, Claus Holst, Eva Prescott, Jørgen Vestbo, Merete Osler. et al. (2002) Smoking reduction, smoking cessation, and mortality: a 16-year follow-up of 19,732 men and women from The Copenhagen Centre for Prospective Population Studies. Am J Epidemiol 156(11): 994-1001.

25. Wannamethee SG, A G Shaper, M Walker, S Ebrahim (1998) Lifestyle and 15-year survival free of heart attack, stroke, and diabetes in middleaged British men. Arch Intern Med 158(22): 2433-2440.

Your next submission with Juniper Publishers
will reach you the below assets
- Quality Editorial service
- Swift Peer Review
- Reprints availability
- E-prints Service
- Manuscript Podcast for convenient understanding
- Global attainment for your research
- Manuscript accessibility in different formats
( Pdf, E-pub, Full Text, Audio)
- Unceasing customer service
Track the below URL for one-step submission
https://juniperpublishers.com/online-submission.php

